\title{
Research on Development of Simulation Interface of Engine Speed Characteristics Based on GT-POWER
}

\author{
Wang Pu-kai, Shi Chao-fan*, Kang Qi, Dong Yi, Han Li-jun \\ Department of Vehicle Engineering, Academy of Armored Forces, Beijing, China \\ Email address: \\ 63962510@qq.com (Shi Chao-fan) \\ ${ }^{*}$ Corresponding author
}

\section{To cite this article:}

Wang Pu-kai, Kang Qi, Dong Yi, Han Li-jun, Shi Chao-fan. Research on Development of Simulation Interface of Engine Speed Characteristics Based on GT-POWER. Science Discovery. Vol. 6, No. 5, 2018, pp. 332-337. doi: 10.11648/j.sd.20180605.14

Received: June 5, 2018; Accepted: September 12, 2018; Published: September 18, 2018

\begin{abstract}
The engine speed characteristic simulation operation interface based on GT-POWER was developed by using VB6.0. VB has the characteristics of simple operation, high efficiency and powerful function. It can realize the background data modification, operation control and calculation result reading and storage of GT-Power model, and draw the engine speed characteristics after finishing the data. curve. Based on ACCESS database, the automatic input and storage of interface input data is realized. The data access, operation control and result reading of GT-POWER model are realized based on VB6.0 programming. The engine is validated by VB6.0 "PictureBox" control. Power, effective torque and effective ratio fuel consumption curve. For the needs of engine speed characteristic simulation, VB6.0 software is used for secondary development of GT-Power, and Chinese operation interface is obtained to simplify the operation process of engine speed characteristic simulation, reduce operation difficulty and improve calculation efficiency, which has certain engineering significance. The simulation method has the advantages of high flexibility, low cost, strong repeatability, and the like, and can obtain parameters that are inconvenient to measure. The results show that the developed engine speed characteristic simulation operation interface can simplify the operation process of engine speed characteristic simulation, reduce the operation difficulty and improve the calculation efficiency.
\end{abstract}

Keywords: Engine, Speed Characteristics, Simulation

\section{基于GT-POWER的发动机速度特性仿真操作界面开发研究}

\author{
王普凯, 石超凡*，康琦，董意，韩立军
}

车辆工程系, 陆军装甲兵学院, 北京, 中国

\section{邮箱}

63962510@qq.com（王普凯）

\begin{abstract}
摘要：采用VB6.0开发了基于GT-POWER的发动机速度特性仿真操作界面。VB具有操作简单、运行高效、功能强大的 特点, 利用它可以实现GT-Power模型的后台数据修改、运行控制和计算结果的读取与储存, 并通过读取的数据进行整 理后绘制发动机速度特性曲线。基于ACCESS数据库实现了界面输入数据的自动读取与存储, 基于VB6.0编程实现了 GT-POWER模型的数据访问、运行控制和结果读取, 利用VB6.0的“PictureBox”控件实现了发动机有效功率、有效扭矩 和有效比油耗曲线的绘制。针对发动机速度特性仿真的需要，使用VB6.0软件对GT-Power进行二次开发，获得中文操 作界面, 以简化发动机速度特性仿真的操作过程、降低操作难度、提高计算效率，具有一定的工程意义。仿真法具有 灵活度高、成本低、可重复性强等优点, 而且可以获取实验不便测取的参数。结果表明, 所开发的发动机速度特性仿 真操作界面, 可以简化发动机速度特性仿真的操作过程、降低操作难度、提高计算效率。
\end{abstract}


关键词: 发动机, 速度特性, 仿真

\section{1. 引言}

发动机的速度特性 [1-4]是指发动机的输出功率、输 出转矩与转速和供油拉杆位移之间的相互关系。通过研究 发动机速度特性, 可以了解发动机动力性、燃油经济性参 数在供油量一定时随发动机转速的变化, 总体掌握发动机 的性能。

研究发动机的速度特性通常有两种方法, 实验法[5-9] 和仿真法[10-13]。实验法工作量大，费用昂贵，而且由于 实际研究中存在许多复杂因素和受实验条件限制, 易造成 较大误差。仿真法具有灵活度高、成本低、可重复性强等 优点, 而且可以获取实验不便测取的参数。仿真法通常通 过软件建立发动机工作过程计算模型, 利用微分方程计算 得到所需数据。

GT-Power采用现代计算机技术和数值计算方法对发 动机性能进行仿真模拟 [14-18], 它功能强大、参数灵活、 结果可靠, 但是采用英文界面, 且操作使用复杂, 对操作 者有较深的专业要求。VB具有操作简单、运行高效、功 能强大的特点, 利用它可以实现GT-Power模型的后台数据 修改、运行控制和计算结果的读取与储存, 并通过读取的 数据进行整理后绘制发动机速度特性曲线。

针对发动机速度特性仿真的需要，使用VB6.0软件对 GT-Power进行二次开发, 获得中文操作界面, 以简化发动 机速度特性仿真的操作过程、降低操作难度、提高计算效 率, 具有一定的工程意义。

\section{2. 发动机速度特性仿真操作界面开发}

利用VB6.0实现对基于GT-POWER的发动机工作过 程模型的二次开发, 所得到的发动机速度特性仿真操作主 界面如图1所示。利用该操作界面, 可以实现：界面数据 的自动读取与存储; 基于 GT-POWER的发动机工作过程仿 真模型的数据修改与运行控制; 基于GT-POWER的发动机 工作过程仿真结果的数据读取; 有效功率、扭矩和比油耗 曲线的自动绘制。

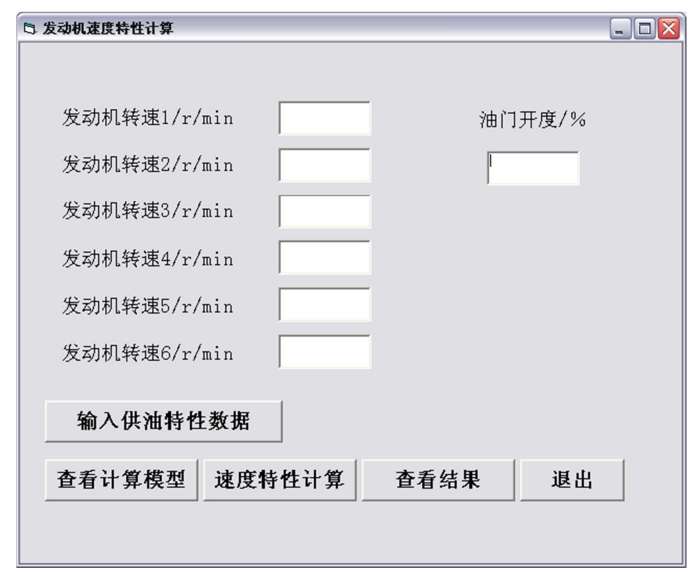

图1 发动机速度特性仿真操作主界面。

\section{1. 界面数据的自动读取与存储}

利用VB6.0的可视化数据管理器, 采用基于ACCESS 数据库的 $\mathrm{VB}$ 编程方法实现界面数据的自动读取与存储, 具体过程为:

1)点击VB6.0软件 “外接程序”菜单栏中的“可视化数 据管理器”选项, 打开VB软件的“可视化数据管理器”, 如 图2所示。

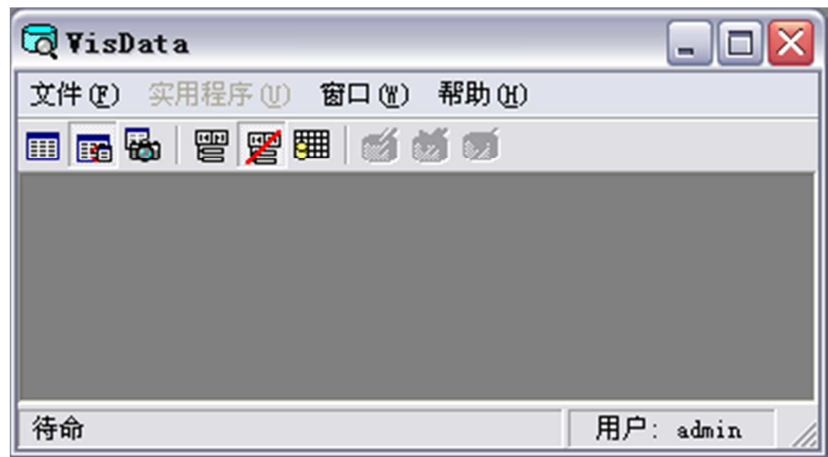

图2 VB软件的可视化数据管理器。

2) 点击 “可视化数据管理器”的 “文件”- “ 新 建”-“"Microsoft Access”-“"Version 7.0 MDB”菜单项, 并 根据提示选择要创建的ACCESS数据库, 如图3所示。

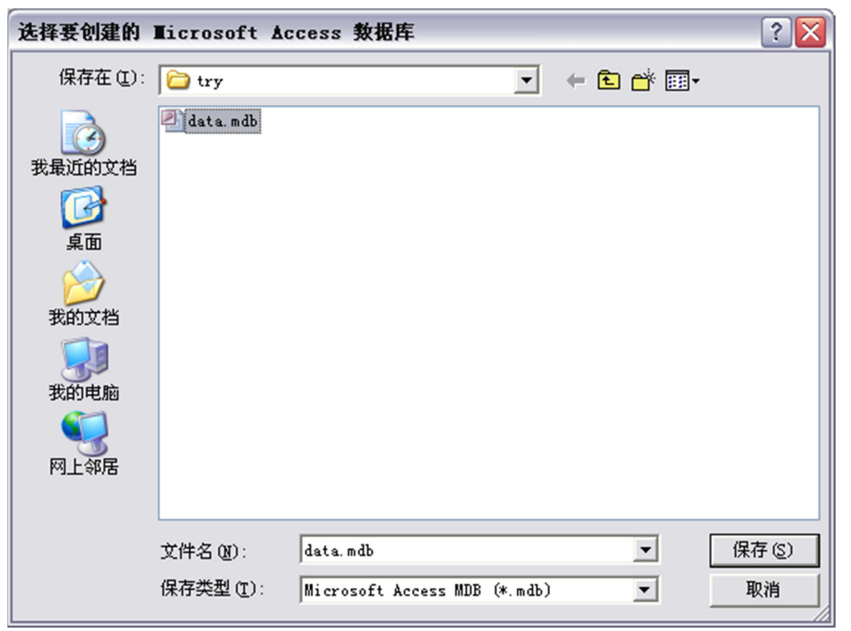

图3 数据库创建过程示意图。

3)点击“可视化数据管理器”的“文件”- “打开数据 库”-“Microsoft Access”菜单项，根据提示打开所建立的 ACCESS数据库,如图4所示。 


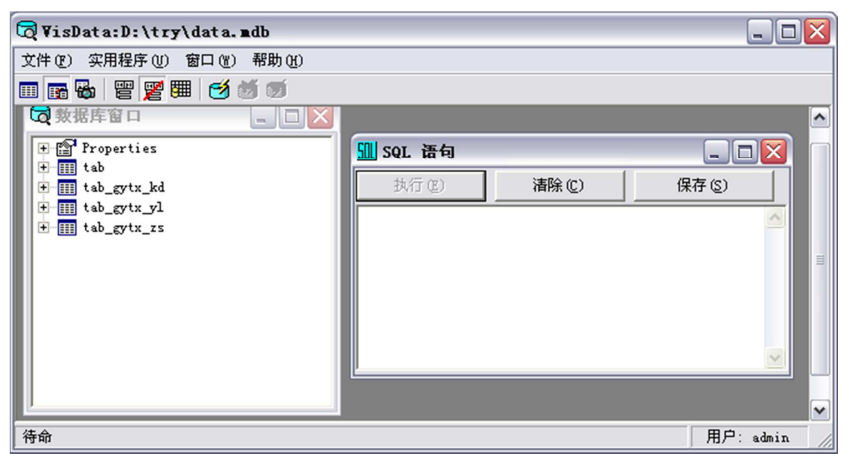

图4 所创建的ACCESS数据库。

4) 鼠标右击 “可视化数据管理器”的数据库窗口中的 “Properties”—“新建表”菜单项, 在弹出的“表结构”界面中 定义ACCESS数据表格名称及表格中各字段的名称、类型 和大小, 如图5所示。

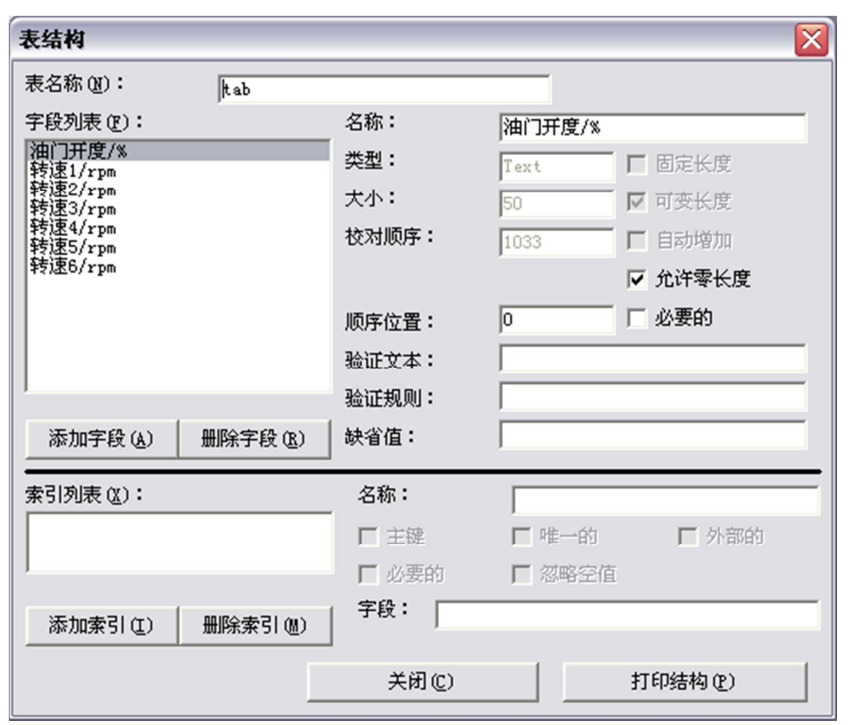

图5 所创建的ACCESS数据库表格。

5)将VB的“Data”控件拖入数据输入操作界面, 然后在 其属性窗口的 “DatabaseName” 项中选择需要关联的 ACCESS数据库, 在“RecordSource”项中选择需要关联的 ACCESS数据表格, 以实现“Data”控件与ACCESS数据库 的数据关联, 如图6所示; 设置数据输入操作界面中各 “text” 控件的属性, 包括“DataSource”和“DataField”项, 以实现 界面输入参数与 “Data”控件的数据关联, 如图7所示。

经过如上 5 步操作, 即可实现界面输入数据在后台 ACCESS数据库中的自动读取与存储。

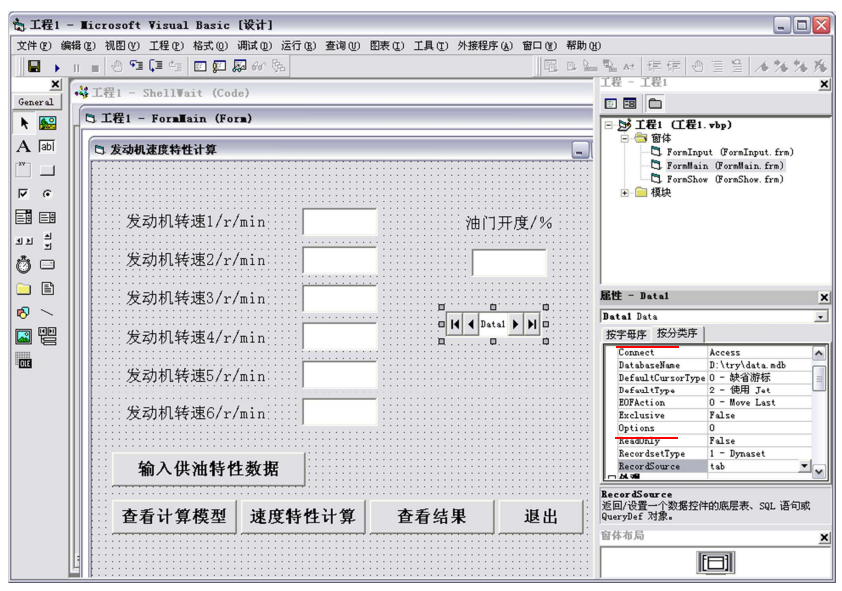

图6 "Data”控件与ACCESS数据库的数据关联。

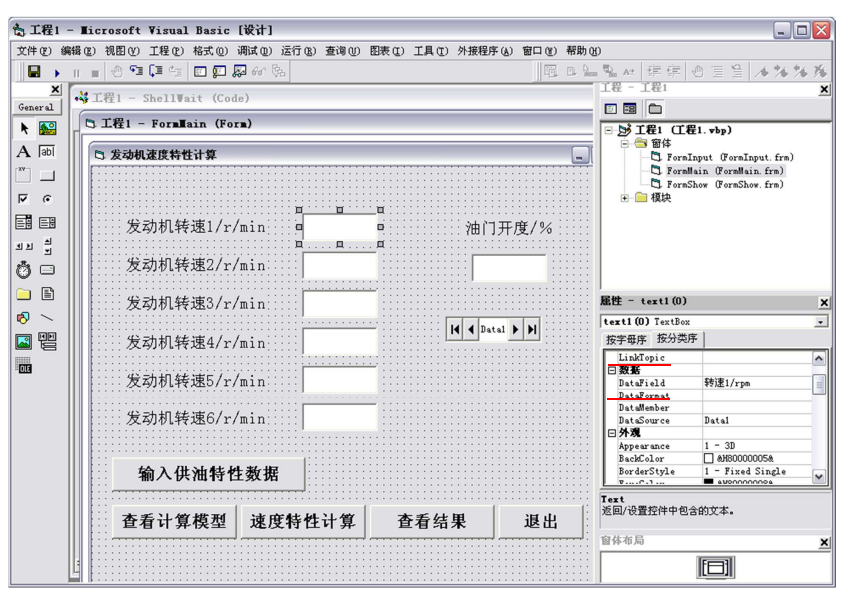

图7 界面输入数据与“Data”控件的数据关联。

\section{2. 仿真模型的数据修改与运行控制}

基于GT-POWER建立发动机工作过程模型, 第一次运 行之后会产生后台数据文件, 其中包含模型结构、部件尺 寸、发动机工况、环境条件等各种数据信息, 如图8所示。

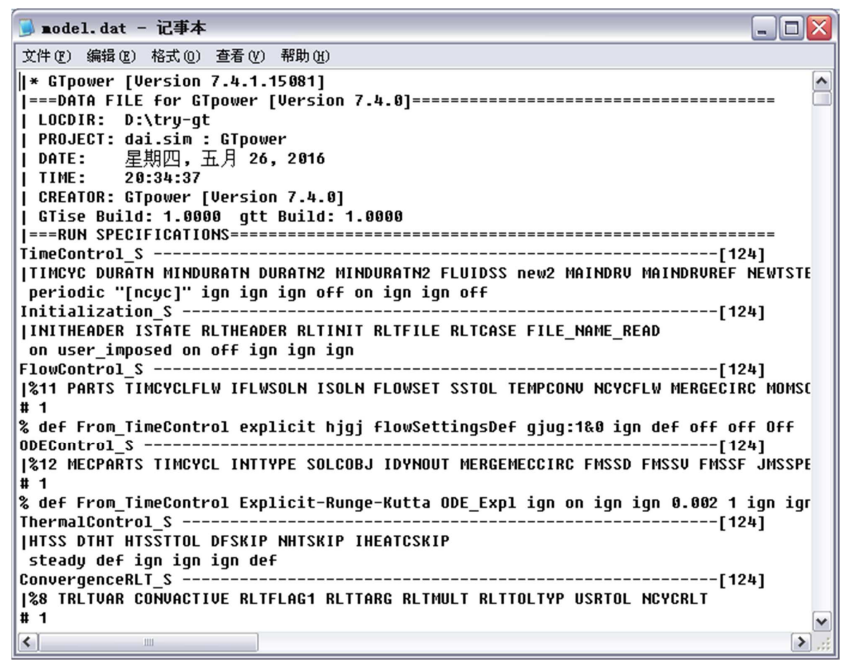

图8 GT-POWER模型后台数据文件。 
基于 GT-POWER所建立的发动机工作过程模型仿真 发动机速度特性时, 需要改写发动机转速和单缸循环供油 量, 方法利用VB6.0编写字符串查找替换程序, 如下所示:

Private Sub ChangeGt(just As Integer, id As String, ok As String)

FileNumber $1=$ FreeFile

Open "model.dat" For Input As \#FileNumber1

FileNumber $2=$ FreeFile

Open "model temp.dat" For Output As \#FileNumber2

Dim textline Ass String

Dim mark As Integer

mark $=0$

Do Until EOF(FileNumber1)

Line Input \#FileNumber1, textline

If textline $<>$ id And mark $=0$ Then Print \#FileNumber2, textline

Else '找到开始改值的地方 mark $=$ mark +1

'更换转速

If just $=1$ Then

If mark $=6$ Then

Print \#FileNumber2, ok

Else End If

Print \#FileNumber2, textline

End If

'更换油量

If just $=2$ Then

If mark $=4$ Then

Else

Print \#FileNumber2, ok End If

Print \#FileNumber2, textline

End If

\section{End If}

Loop

Close \#FileNumber1

Close \#FileNumber2

Kill "model.dat"

Name "model_temp.dat" As "model.dat"

End Sub

利用VB6.0编写进程控制程序, 如下所示:

Sub ShellWait(cCommandLine As String)

Dim hShell As Long

Dim hProc As Long

Dim IExit As Long

hShell $=$ Shell(cCommandLine, vbNormalFocus)

$\mathrm{hProc}$

OpenProcess(PROCESS_QUERY_INFORMATION, False, hShell)

Do

GetExitCodeProcess hProc, lExit

DoEvents

Loop While 1Exit $=$ STILL_ACTIVE

End Sub

采用如下语句实现GTPOWER后台计算引擎对所建 立的GT-POWER模型的调用计算:

ShellWait ("C:IProgram Files\GTIbin \GTpower.bat model.dat")

\section{3. 仿真结果的数据读取}

GT-POWER计算结果存放于“.out”文件中, 如图9所示。 利用VB6.0编程、采用查找并读取字符串的方法读取有效 功率, 根据已知的发动机转速和读取的有效功率换算得到 有效扭矩; 根据发动机转速、气缸数目、单缸循环供油量 和有效功率计算得到有效比油耗。

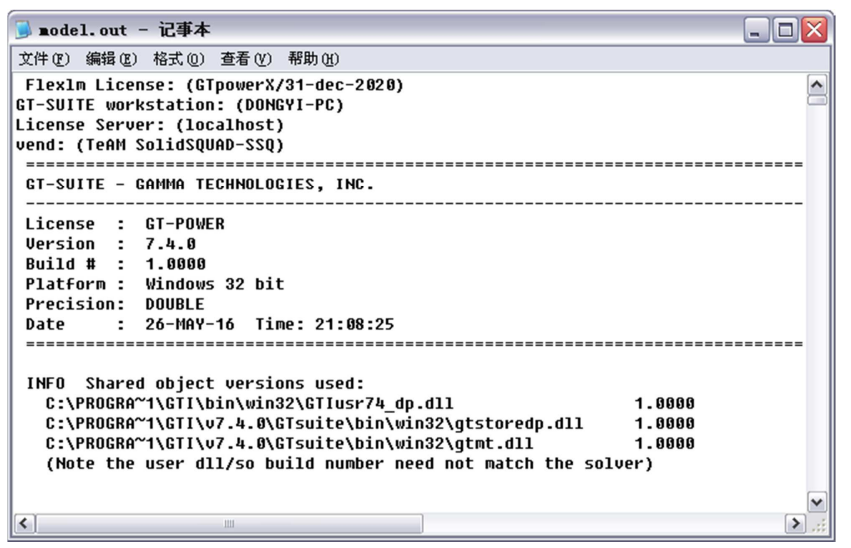

图9 GT-POWER计算结果文件。

对于四行程发动机，其有效扭矩和有效比油耗计算公 式如下:

$$
\begin{array}{r}
M_{\mathrm{e}}=1000 N_{\mathrm{e}} /\left(n_{\mathrm{e}} \cdot \frac{\pi}{30}\right) \\
g_{\mathrm{e}}=3.6\left(i b_{\mathrm{e}} n_{\mathrm{e}} / 120\right) / N_{\mathrm{e}}
\end{array}
$$

式中: $M_{\mathrm{e}}$ 为有效扭矩, $\mathrm{N} \cdot \mathrm{m} ; g_{\mathrm{e}}$ 为有效比油耗, $\mathrm{g} / \mathrm{h} / \mathrm{kW} ; N_{\mathrm{e}}$ 为有效功率, $\mathrm{kW} ; n_{\mathrm{e}}$ 为发动机转速, $\mathrm{r} / \mathrm{min}$; $b_{\mathrm{e}}$ 为单缸循环供油量, $\mathrm{mg} ; i$ 为气缸数目。

\section{4. 仿真结果的曲线绘制}

利用VB6.0编写程序实现对同油门开度下各转速点的 一次性计算, 各转速下的有效功率、有效扭矩、有效比油 耗计算结果成一行依次保存于“Result.dat”文件中, 如图10 所示, 利用VB6.0的“PictureBox”控件绘制速度特性曲线, 部分语句如下:

\section{'利用 $x y$ ( ) 数据绘制二维曲线}

Pic1.Scale $(-1,11)-(11,-1)$ '定义坐标系原点靠近 左下角

Pic1.Line $(-1,0)-(10,0)$ '画X轴

Pic1.Line $(0,-1)-(0,10)$ '画Y轴

'画X轴刻度

For $\mathrm{i}=-1$ To 10 Step 1

$$
\begin{aligned}
& \text { If } \mathrm{i}<>0 \text { Then } \\
& \text { Pic1.Line (i, 0)-(i, 0.2) }
\end{aligned}
$$
End If

Next

'画Y轴刻度

For $\mathrm{j}=-1$ To 10 Step 1 


$$
\text { If } \mathrm{j}<>0 \text { Then }
$$

End If

Pic1.Line $(0, \mathrm{j})-(0.2, \mathrm{j})$

Next

For $\mathrm{M}=1$ To $(\mathrm{k}-1)$

'画数据曲线

Pic1.Line (xy(M - 1, 0), xy(M - 1, 1))-(xy(M,

$0), x y(M, 1)), \operatorname{RGB}(255,0,0)$

'标注工况点

Pic1.Current $X=x y(M-1,0)$

Pic1.CurrentY $=x y(M-1,1)$

Pic1.Print "+"

Next

Pic1.Current $X=x y(k-1,0)$

Pic1.Current $Y=x y(k-1,1)$

Pic1.Print "+"

\section{3. 仿真实例} 型。

基于GT-POWER建立的某型发动机工作过程计算模

速度特性仿真计算界面输入参数如图10和图11所示。

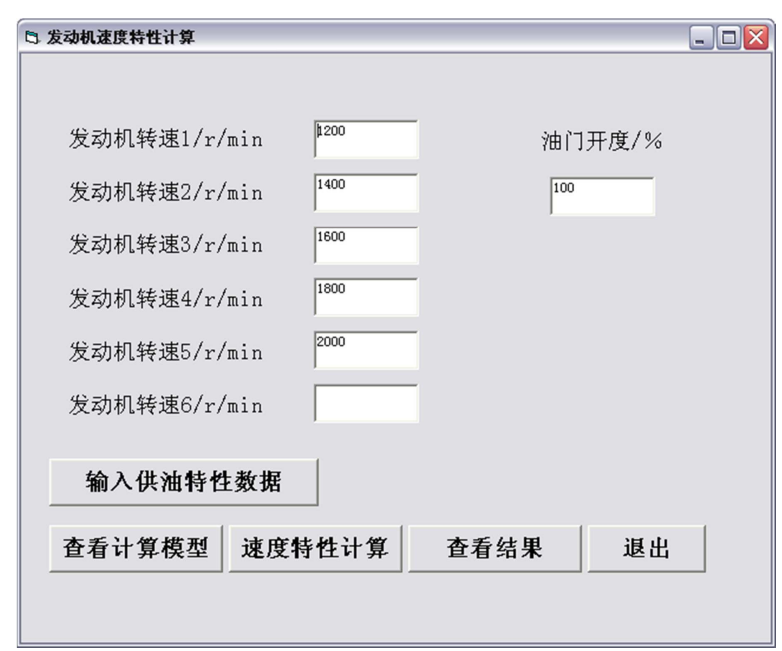

图10 计算输入的发动机转速和油门开度数据。

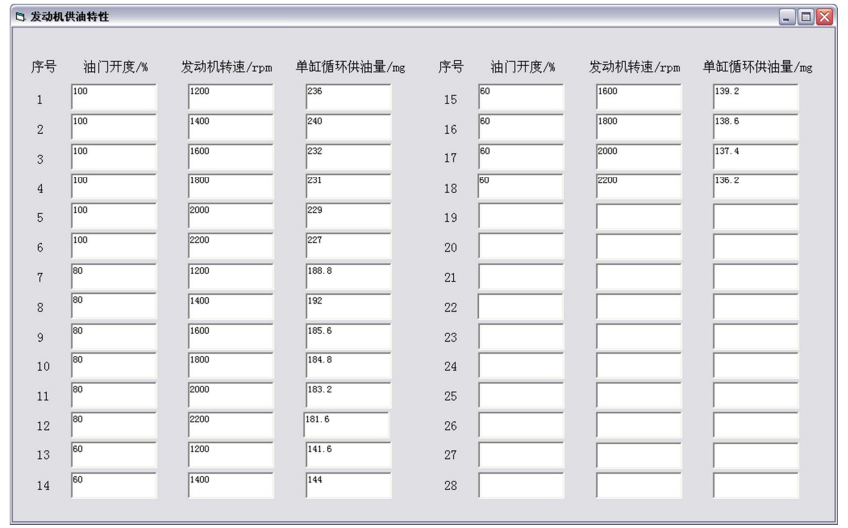

图11 计算输入的发动机供油特性数据。

点击主操作界面中的“查看计算模型”按钮, 可直接调 用GT-POWER打开所建立的发动机工作过程计算模型; 点
击“速度特性计算”启动GT-POWER后台计算引擎、调用所 建立的发动机工作过程模型进行计算, 如图12所示。

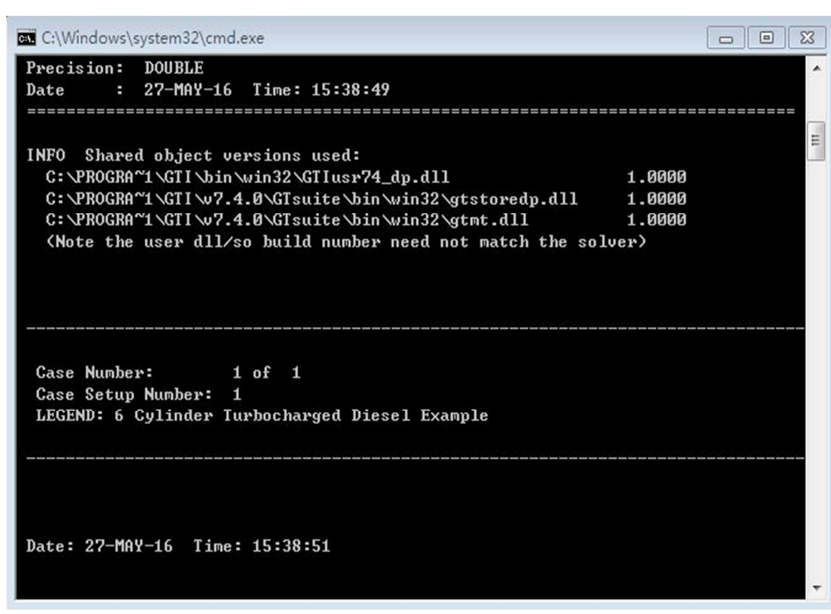

图12 发动机工作过程计算界面。

计算完成后, 点击“查看结果”按钮, 弹出结果显示界 面, 在其中选择需要显示的曲面, 如图13 图15所示。

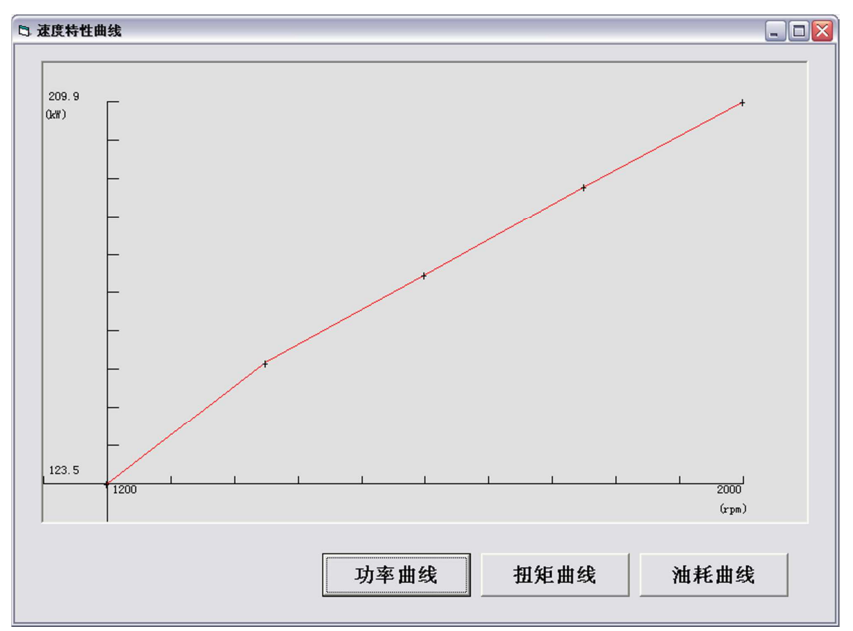

图13 发动机有效功率仿真曲线。

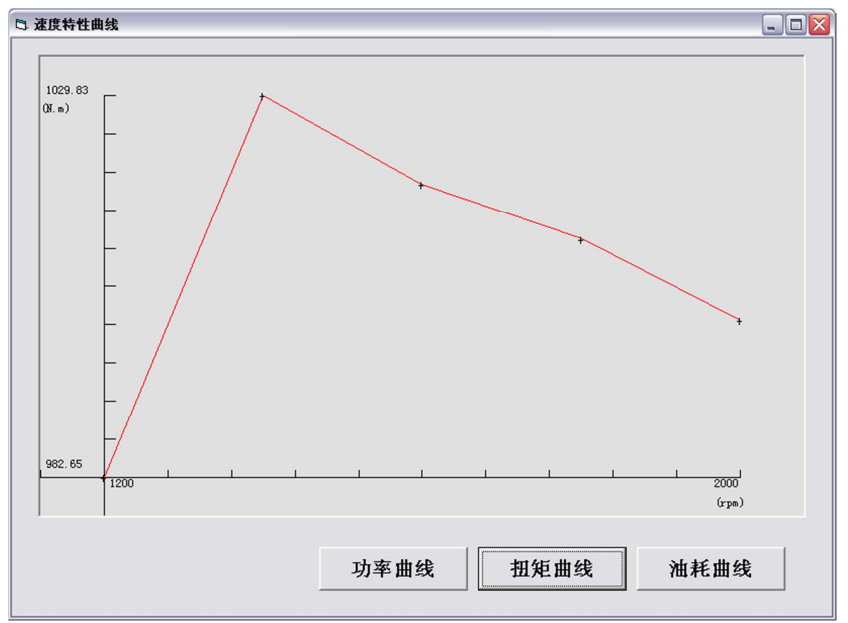

图14 发动机有效扭矩仿真曲线。 


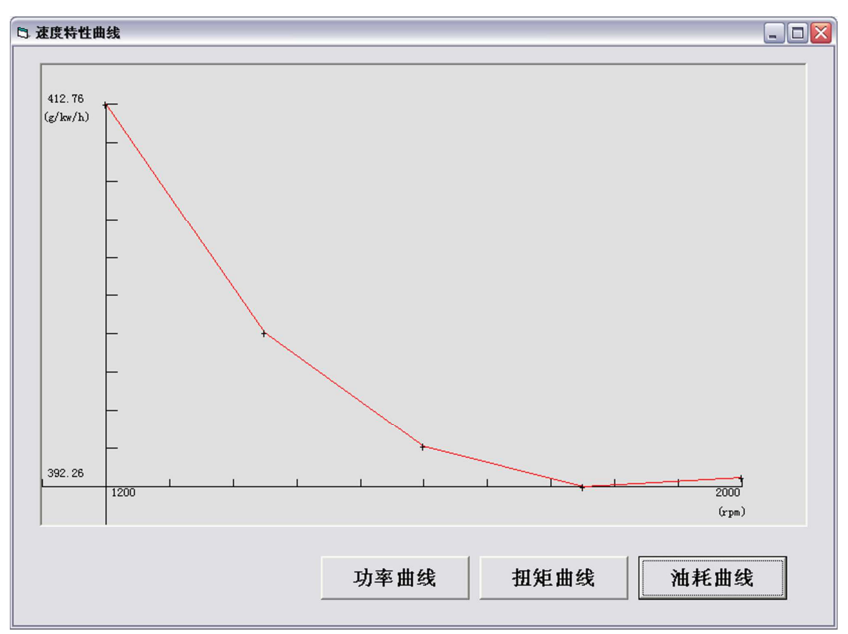

图15 发动机有效比油耗仿真曲线。

\section{4. 结论}

采用VB6.0开发了基于GT-POWER的发动机速度特 性仿真操作界面。基于ACCESS数据库实现了界面输入数 据的自动读取与存储, 基于VB6.0编程实现了 GT-POWER 模型的数据访问、运行控制和结果读取, 利用VB6.0的 “PictureBox”控件实现了发动机有效功率、有效扭矩和有 效比油耗曲线的绘制。结果表明, 所开发的发动机速度特 性仿真操作界面, 可以简化发动机速度特性仿真的操作过 程、降低操作难度、提高计算效率。

\section{参考文献}

[1] 王宪成, 张更云, 韩树等.车用内燃机学 $[\mathrm{M}]$. 北京: 兵器工 业出版社,2006:145。

[2] 左承基,欧阳明高,刘铮.柴油机-天然气双燃料发动机工作特 性分析[J].石油机械,1999,27（8）:16-18。

[3] 于秀敏,唐睿,杨世春,刘乐.汽油机与LPG发动机冷起动特性 试验[J].农业机械学报,2007,38（4）:4-7。
[4] 梁玲,周经渊.发动机速度特性的数学模型 $[\mathrm{J}]$.农长沙铁道学 院学报,1995,13（3）:48-51。

[5] 朱伟伟.发动机特性建模方法的选择[J].现代车用动力,2007 (2) :27-30。

[6] 赵亚男,赵福堂,刘碧荣.汽车发动机特性仿真研究[J].北京交 通大学学报,2008, 32 (1):97-100。

[7] 张永栋.基于GT-Power柴油机性能仿真分析[J].机电工程技 术,2010,39（10）:38-39,98。

[8] 王娟,李明海.GT-Power在机车柴油机上的仿真应用[J].长沙 交通学院学报,2008, 24（3）:76-80。

[9] 白杰,杨毅成,王伟. 航空发动机防火试验火焰特征数值仿真 分析[J].燃气浴轮试验与研究, 2017,30(5):1-7。

[10] 周晓斌. 发动机配气机构振动噪声仿真研究 [D]. 重庆大 学,2016。

[11] 李素华.变转速条件下汽车发动机故障模式识别仿真 [J].计 算机仿真,2016,33(11):144-147。

[12] 李金杨赪石,彭博,等.基于联合仿真技术的转缸式斜盘活塞 发动机动力学研究 [J].船舶工程, 2017(8):35-39。

[13] 纪文晓.缸内直喷 CNG发动机稀薄燃烧特性仿真研究[D].重 庆交通大学, 2016。

[14] 杜祥哲.微型斯特林发动机的设计与仿真 [D].东北林业大 学, 2016。

[15] 向熔.高压共轨四缸柴油机振动与噪声特性及影响因素研 究[D].昆明理工大学, 2016。

[16] 吕赫.废气再循环对氢气汽油双燃料发动机性能影响的仿 真研究[D].吉林大学,2017。

[17] 白锦洋, 吴学雷, 高峰, 等. 多轴车辆动力传动系统建模与仿真 [J].北京航空航天大学学报,2017,43(1):136-143。

[18] 杜子学,沈宏丽,叶双平,等.某型SUV车发动机舱热管理仿真 分析及优化 $[\mathrm{J}]$. 小型内燃机与车辆技术, $2016,45(2): 48-51$ 。 